\title{
Therapeutic Drugs Targeting 2019-nCoV Main Protease by High-Throughput
}

\section{Screening}

Yan $\mathrm{Li}^{1}{ }^{1 \#}$, Jinyong Zhang ${ }^{2 \#}$, Ning Wang ${ }^{1}$, Haibo $\mathrm{Li}^{2}$, Yun $\mathrm{Shi}^{1}$, Gang Guo ${ }^{1}$, Kaiyun $\mathrm{Liu}^{1}$, Hao Zeng ${ }^{2 *}$, Quanming Zou ${ }^{2 *}$

${ }^{1}$ West China Biopharm Research Institute, West China Hospital, Sichuan University, Chengdu, Sichuan, 610041, PR China.

${ }^{2}$ National Engineering Research Center of Immunological Products, Department of Microbiology and Biochemical Pharmacy, College of Pharmacy, Army Medical University, Chongqing,400038, PR China.

${ }^{\#}$ These authors contribute equally to this paper.

*Corresponding author: Hao Zeng (zeng1109@163.com),

Quanming Zou (qmzou2007@163.com) 


\begin{abstract}
2019 Novel Coronavirus (2019-nCoV) is a virus identified as the cause of the outbreak of pneumonia first detected in Wuhan, China. Investigations on the transmissibility, severity, and other features associated with this virus are ongoing. Currently, there is no vaccine or therapeutic antibody to prevent the infection, and more time is required to develop an effective immune strategy against the pathogen. In contrast, specific inhibitors targeting the key protease involved in replication and proliferation of the virus are the most effective means to alleviate the epidemic. The main protease of SARS-CoV is essential for the life cycle of the virus, which showed $96.1 \%$ of similarity with the main proteaseof $2019-\mathrm{nCoV}$, is considered to be an attractive target for drug development. In this study, we have identified 4 small molecular drugs with high binding capacity with SARS-CoV main protease by high-throughput screening based on the 8,000 clinical drug libraries, all these drugs have been widely used in clinical applications with guaranteed safety, which may serve as promising candidates to treat the infection of 2019-nCoV.
\end{abstract}

Keywords: 2019-nCoV; SARS-CoV; main protease; therapeutic drugs. 


\section{Introduction}

Since December 2019, a series of pneumonia cases of unknown cause emerged in Wuhan, China. Genome sequencing of samples from these patients confirmed that the culprit of these infections was a beta-coronavirus that has never been reported before, which was later named as 2019-nCoV [1,2]. By Jan 28, 2020, more than 4500 patients were confirmed to be infected with 2019-nCoV, and nearly 100 patients were died from the infection. So far, the infection keeps spreading and more and more exported cases were confirmed in other provinces in China, and several other countries, including the United States, posing great pressure on public health security.

The discovery and clinical application of specific drugs against 2019-nCoV is an effective means to alleviate the current epidemic. However, there are no clinically effective drugs for this virus for the moment. Although 2019-nCoV is significantly different from SARS-CoV, which breakout in Beijing 17 years before [3], the sequence identity between themis as high as $79.5 \%$. Further sequence alignment revealed that the similarity of the sequence of the main protease between $2019-\mathrm{nCoV}$ and SARS-CoV is up to $96.1 \%$. Previous study demonstrated that the main protease of SARS-CoV is essential for the life cycle of the virus, and is considered to be an attractive target for drug development [4]. Thus, this protein could be used as a homologous target to screening drugs that inhibiting the replication and proliferation of 2019-nCoV.

In order to screening the possible drug candidates that were able to prevent or cure the infection, high-throughput screening was performed based on the 8,000 clinical drug libraries using the online software Vina and SeeSAR, combined with our in-house automatic processing scripts and screening programs, and 4 small molecular drugs with high binding capacity with SARS-CoV main protease were identified. 
These drugs were widely used in clinical practice, so the safety was guaranteed. In view of the activity and safety of these wildly used drugs, it is feasible to conduct clinical exploratory treatment in special cases such as the outbreak of 2019-nCoV. Here we report the identify and structure of these molecules.

\section{Material and Methods}

\subsection{Data resources}

The CDS and proteins of Wuhan seafood market pneumonia virus (Accession: NC_045512.2) were obtained from NBCI database. The structures and sequences of SARS-CoV main protease were downloaded from PDB database. Nearly 8000 molecules were obtained from Drugbank [5], including the approval or experimental compounds and small molecules

\subsection{Similarity search}

Molecular similarity search was performed by using a strategy based on the similar sequences ofthe structure-revealed molecules. The NCBI-blast v2.9 was installed in local machine, then a local database was established by the molecular sequences of SARS-CoV main protease from PDB. The blastp was ran by default parameter.

\subsection{Molecular docking}

The AutoDock vina was obtained from website of scripps.edu [6]. The crystal structure of main protease monomer (PDB id: 5n5o) was used as target protein after removing the unrelated complex molecule by AutoDockTools. The receptor was prepared by removing water, adding hydrogen and computing charges. The binding coordinates were located by Grid box including the cave near N-terminal and the joint groove of dimer. Autodocking was performed by using multithread tasks by our in-house script, and potential molecules were screening out by a Perl program 
developed by us.

\subsection{Protein-ligand interaction Analysis}

The candidates harvested from docking were deeply analyzed for atom-based affinity contributions and physical-chemical properties by SeeSAR (version 9.2; BioSolveIT GmbH, Sankt Augustin, Germany, 2019, www.biosolveit.de/SeeSAR).

\subsection{Structure Display}

The 3D molecule images were displayed by PyMOL v2.3 [7].

\subsection{Screening criteria}

The affinities of Vina less than $-7.7 \mathrm{kcal} / \mathrm{mol}$ were harvested initially. Then those molecules were removed such as toxin, experimental and unapproved ones, and those with strong side effects. Finally, the candidate drugs were selected with better affinities in Vina and SeeSAR.

\section{Results}

\subsection{Homologous targets screening}

We downloaded the sequences of a total of 103 SARS-CoV main proteases with different origins from PDB and built a local library for homology alignment. The result revealed that the similarity between the region at $3264 \sim 3570$ aa of $2019-\mathrm{nCoV}$ ORF1 ab protein and SARS-CoV main protease 5n5o was up to $96.1 \%$ (Fig. 1). Thus, the main protease is highly conserved and main protease $5 \mathrm{n} 5$ ocan be used as a homologous target for screening of possible drugs against 2019-nCoV.

\subsection{Binding sites of target protein detection}

There were three binding pockets detected in the viral main protease by computation, as labeled in Fig.2C. In comparison to the dimer and monomer structure of the protein (Fig.2A and B), we could figure out that the pocket 1 was the binding site with natural substrate, pocket 2 was the joint groove between two monomers, and 
a small pocket 3 was located in the $\mathrm{C}$ terminal.

\subsection{Candidates summary}

At first, we harvested 690 molecules with possible binding capacity with SARS-CoV main protease, but most of them were dyes, toxins and antitumor drugs with strong side effects, about 50 molecules were left after excluding these molecules and other neurologic drugs, and then we selected marketable drugs from them for further kinetic and bio-chemical analysis. Finally, 4 molecules were identified, including No. 6651 molecule (Prulifloxacin), No. 6589 molecule (Bictegravir), No. 0097 molecule (Nelfinavir) and No. 6626 molecule (Tegobuvi).

\subsection{The interaction between Prulifloxacin and viral protease}

The binding energies of No. 6651 molecule (Prulifloxacin, Fig. 3A) to viral main protease were $-8.2 \mathrm{Kcal} / \mathrm{mol},-8.2 \mathrm{Kcal} / \mathrm{mol},-7.9 \mathrm{kcal} / \mathrm{mol}$ at three binding sites, respectively (Fig. 3B), which were the cave adjacent to the $\mathrm{N}$-terminal, the dimer joint groove and its back side. Moreover, at several docking poses, all of the estimated affinitiesof binding were between $\mu \mathrm{M}$ to $\mathrm{mM}$. The evaluations of ligand-lipophilicity efficiency (LLE), torsion and clash were also satisfied.

\subsection{The interaction between Bictegravir and main protease}

The binding sites between Bictegravir and the main protease was displayed at Fig. $4 \mathrm{~B}$, the binding energy between Bictegravir and major protease was $-8.3 \mathrm{kcal} / \mathrm{mol}$ at the joint groove, but only $-7.3 \mathrm{kcal} / \mathrm{mol}$ at the active site, and the estimated affinity is satisfied (Fig. 4C).

\subsection{The interaction between Nlfinavir and main protease}

The results showed that the binding site between Nelfinavir and the main protease was located at the joint groove (Fig. 5B). The binding energy between Nelfinavir and major protease was $-8.6 \mathrm{kcal} / \mathrm{mol}$, but the estimated affinity seemed 
not high enough (Fig. 5C).

\subsection{The interaction between Tegobuvir and main protease}

Tegobuvir was docked into the joint groove of main protein, the binding energy between them was $-8.9 \mathrm{kcal} / \mathrm{mol}$, and the estimated affinity is satisfied (Fig. 6C). The binding sites was displayed at Fig. 6B.

\section{Discussion}

Although coronaviruses are under extensive mutagenesis, some key proteins, especially replication-related enzymes, are highly conserved [8]. Because the mutation in key proteins are often lethal to the virus [9], drugs that target conservative protease are usually capable of preventing the replication and proliferation of the virus and exhibit broad spectrum. Besides, they can reduce the risk of mutation mediated drug-resistance.

In the current study, based on the results from bioinformatics analysis, the structure of SARS-CoV 5n5o protein was selected as a homologous target for molecule screening. Then, in silico high throughput screening strategy and automatic pipeline have been established by using classic docking software and our in-house program, which greatly accelerates the screening process.

Among the four molecules identified in this study, Prulifloxacin is a chemotherapeutic fluoroquinolone antibiotic with broad-spectrum activity, it has been approved for the treatment of uncomplicated and complicated urinary tract infections, community-acquired respiratory tract infections in Italy and gastroenteritis, including infectious diarrheas, in Japan [10]. Unfortunately, Prulifloxacin is reported as a prodrug, which is rapidly metabolized to ulifloxacin in vivo [11]. But it can be used as a lead compound that provide guidance for our later structural modification and optimization to design more effective main protease inhibitors. Tegobuvir is a novel 
non-nucleoside inhibitor (NNI) of HCV RNA replication with demonstrated antiviral activity in patients with genotype 1 chronic HCV infection [12]. In addition, both of Nelfinavir and Bictegravir are anti-HIV drugs, of which Nelfinavir is a protease Inhibitors inhibit the cleavage of the polyprotein gag-pol [13], whereas Bictegravir is a new and potent HIV-1 integrase inhibitor, which is able to efficiently prevent HIV from multiplying and can reduce the amount of HIV in the body [14].

Our results clearly showed that all the four molecules showed reasonable binding conformations with the viral main protease. Among them, Prulifloxacin exhibited three binding sites with main protease, two of which were the same as Nelfinavir and Bictegravir. Further, molecule Prulifloxacin, Tegobuvir and Bictegravir are preferable to Nelfinaviras confirmed by affinity and physical-chemical properties analysis using SeeSAR. Based on the pockets' functions of target protein, it suggested that these molecules should possess the abilities to block the active sites or interrupt the dimer formation of viral protein. Therefore, they may sever as promising candidates for drug repurpose and development against 2019-nCoV.

\section{Reference}

1. Hui DS, E IA, Madani TA, Ntoumi F, Kock R, et al. (2020) The continuing 2019-nCoV epidemic threat of novel coronaviruses to global health - The latest 2019 novel coronavirus outbreak in Wuhan, China. Int J Infect Dis 91: 264-266.

2. Zhu N, Zhang D, Wang W, Li X, Yang B, et al. (2020) A Novel Coronavirus from Patients with Pneumonia in China, 2019. N Engl J Med.

3. Cooke FJ, Shapiro DS (2003) Global outbreak of severe acute respiratory syndrome (SARS). Int J Infect Dis 7: 80-85.

4. Xia B, Kang X (2011) Activation and maturation of SARS-CoV main protease. Protein Cell 2: 282-290.

5. Wishart DS, Feunang YD, Guo AC, Lo EJ, Marcu A, et al. (2018) DrugBank 5.0: a major update to the DrugBank database for 2018. Nucleic Acids Res 46: D1074-D1082.

6. Trott O, Olson AJ (2010) AutoDock Vina: improving the speed and accuracy of docking with a new scoring function, efficient optimization, and multithreading. J Comput Chem 31: 455-461.

7. Janson G, Zhang C, Prado MG, Paiardini A (2017) PyMod 2.0: improvements in protein 
sequence-structure analysis and homology modeling within PyMOL. Bioinformatics 33: 444-446.

8. Zhou P, Yang X-L, Wang X-G, Hu B, Zhang L, et al. (2020) Discovery of a novel coronavirus associated with the recent pneumonia outbreak in humans and its potential bat origin. bioRxiv: 2020.2001.2022.914952.

9. Zhang D, Chen J, Deng L, Mao Q, Zheng J, et al. (2010) Evolutionary selection associated with the multi-function of overlapping genes in the hepatitis B virus. Infect Genet Evol 10: 84-88.

10. Fritsche TR, Biedenbach DJ, Jones RN (2009) Antimicrobial activity of prulifloxacin tested against a worldwide collection of gastroenteritis-producing pathogens, including those causing traveler's diarrhea. Antimicrob Agents Chemother 53: 1221-1224.

11. Matera MG (2006) Pharmacologic characteristics of prulifloxacin. Pulm Pharmacol Ther 19 Suppl 1: 20-29.

12. Hebner CM, Han B, Brendza KM, Nash M, Sulfab M, et al. (2012) The HCV non-nucleoside inhibitor Tegobuvir utilizes a novel mechanism of action to inhibit NS5B polymerase function. PLoS One 7: e39163.

13. Davis DA, Soule EE, Davidoff KS, Daniels SI, Naiman NE, et al. (2012) Activity of human immunodeficiency virus type 1 protease inhibitors against the initial autocleavage in Gag-Pol polyprotein processing. Antimicrob Agents Chemother 56: 3620-3628.

14. Tsiang M, Jones GS, Goldsmith J, Mulato A, Hansen D, et al. (2016) Antiviral Activity of Bictegravir (GS-9883), a Novel Potent HIV-1 Integrase Strand Transfer Inhibitor with an Improved Resistance Profile. Antimicrob Agents Chemother 60: 7086-7097. 


\section{Figure legends}

Fig. 1. The blast results of ORF1ab of 2019-nCoV against SARS-CoV main proteases.

Fig. 2. Binding pockets and functional sites of SARS-CoV main protease A: Dimer structure; B: Monomer structure; C: Detected pockets.

Fig.3. 2D molecular structure of Prulifloxacin (A); Binding conformation of Prulifloxacin to viral protease (B); Physicochemical featureand affinity of Prulifloxacin to viral protease (C).

Fig. 4. 2D molecular structure of Bictegravir (A); Binding conformation of Bictegravir to viral protease (B); Physicochemical featureand affinity of Bictegravir to viral protease $(\mathrm{C})$.

Fig. 5. 2D molecular structure of Nelfinavir (A); Binding conformation of Nelfinavir to viral protease (B); Physicochemical feature and affinity of Nelfinavir to viral protease $(\mathrm{C})$.

Fig.6. 2D molecular structure of Tegobuvir (A); Binding conformation of Tegobuvir to viral protease (B); Physicochemical feature and affinity of Tegobuvir to viral protease (C) 
Databanat caln pretena

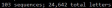

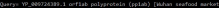

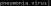

Letehingas

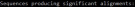

$5 \cos$

(sit)!

$+$

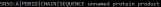

81

(4)

esi

s.1

Valun

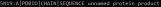

471

ded

sil

41

에

0.9

A. 사

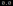

, 9.9

A.

Q.

ens

B.

8.0

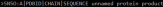

Levition

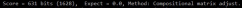

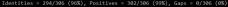

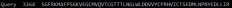

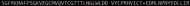

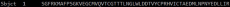
3927

29

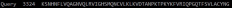
syas

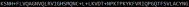

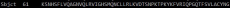

1 in

Questr.

3204

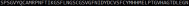

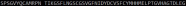

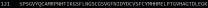

baft aris

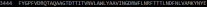

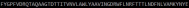

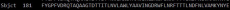

sati

2 and

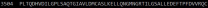
.3855

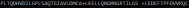

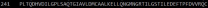

Qustp 3664 sinted 3 sia seving

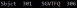




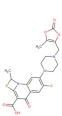

\section{C}

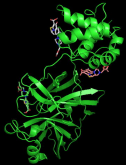

pitatis

wher

* $\mathrm{k} \times$

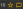

in 1 in

$4 x \rightarrow=$

1 it $=$

1.

17 is: $=$

a $x=10$

$x+\frac{1}{2}$

$x+2=$

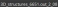

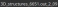

20 -

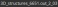

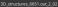

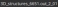

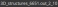

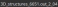

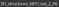

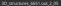

$-\frac{1}{4}$

v

(5)

$\boldsymbol{v}$

$\sqrt{*}$

ㄷ.7

v

v

8

$\sigma$

$\mathbf{v}$ in

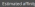
wat ting fas

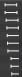

.

2 t.

8

.

2.

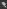

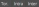
itar igat:

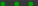


A

B

$\left.\int_{0,2}^{2}+2+1\right]$

C

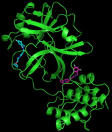

\section{tinn}

갈

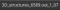

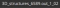

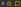

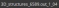

i. 4 A $\mathrm{E}$

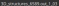

15

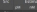

se

viv

c.

er:

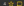


A

B

C

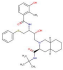

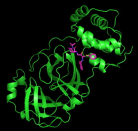

Rime

30. structiras 0207 art 02

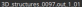

Eic Bar nall

(8)

$\theta$

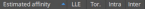
IM nW 3. 3

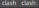



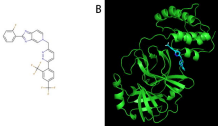

fetse

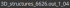

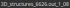

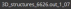

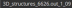
In

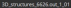

372

e.

(7)

(i)

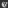

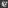

0

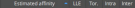
N sist

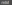

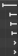

Wast $140,+$

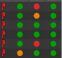

